$1-1-2014$

\title{
Fidelity threshold of the surface code beyond single-qubit error models
}

Pejman Jouzdani

University of Central Florida

E. Novais

I. S. Tupitsyn

Eduardo R. Mucciolo

University of Central Florida

Find similar works at: https://stars.library.ucf.edu/facultybib2010 University of Central Florida Libraries http://library.ucf.edu

This Article is brought to you for free and open access by the Faculty Bibliography at STARS. It has been accepted for inclusion in Faculty Bibliography 2010 s by an authorized administrator of STARS. For more information, please contact STARS@ucf.edu.

\section{Recommended Citation}

Jouzdani, Pejman; Novais, E.; Tupitsyn, I. S.; and Mucciolo, Eduardo R., "Fidelity threshold of the surface code beyond single-qubit error models" (2014). Faculty Bibliography 2010s. 5534.

https://stars.library.ucf.edu/facultybib2010/5534

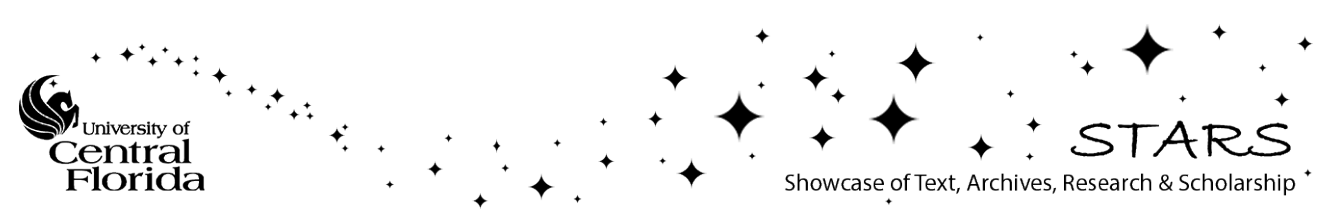




\title{
Fidelity threshold of the surface code beyond single-qubit error models
}

\author{
Pejman Jouzdani, ${ }^{1}$ E. Novais, ${ }^{2}$ I. S. Tupitsyn, ${ }^{3,4}$ and Eduardo R. Mucciolo ${ }^{1}$ \\ ${ }^{1}$ Department of Physics, University of Central Florida, Orlando, Florida 32816, USA \\ ${ }^{2}$ Centro de Ciências Naturais e Humanas, Universidade Federal do ABC, Santo André, São Paulo, Brazil \\ ${ }^{3}$ Physics Department, University of Massachusetts, Amherst, Massachusetts 01003, USA \\ ${ }^{4}$ Pacific Institute of Theoretical Physics, University of British Columbia, Vancouver, British Columbia, Canada V6T 1Z1
}

(Received 3 February 2014; revised manuscript received 30 July 2014; published 13 October 2014)

\begin{abstract}
The surface code is a promising alternative for implementing fault-tolerant, large-scale quantum information processing. Its high threshold for single-qubit errors under stochastic noise is one of its most attractive features. We develop an exact formulation for the fidelity of the surface code that allows us to probe much further on this promise of strong protection. This formulation goes beyond the stochastic single-qubit error model approximation and can take into account both correlated errors and inhomogeneities in the coupling between physical qubits and the environment. For the case of a bit-flipping environment, we map the complete evolution after one quantum error correction cycle onto the problem of computing correlation functions of a two-dimensional Ising model with boundary fields. Exact results for the fidelity threshold of the surface code are then obtained for several relevant types of noise. Analytical predictions for a representative case are confirmed by Monte Carlo simulations.
\end{abstract}

DOI: 10.1103/PhysRevA.90.042315

PACS number(s): 03.67.Lx, 03.67.Pp, 03.65.Yz, 05.50.+q

\section{INTRODUCTION}

Quantum error correction (QEC) is one of the most important tools to reduce the effects of decoherence in quantum systems that process information. Several different protocols have been developed since QEC was first introduced [1], but particular attention has been given to stabilizer codes [2]. Among them, the surface code $[3,4]$ is perhaps the most promising for large-scale implementations [5]. Its main virtues are (i) qubits are disposed in a planar array, only requiring local measurement operations; and (ii) early estimates based on stochastic error models indicate a very large threshold value, $p_{c} \approx 11 \%$ [4], for the single-qubit error probability $p$. For $p<p_{c}$, the probability of successful encoding tends to 1 as the number of physical qubits is increased.

Despite the large theoretical effort that has been devoted to characterizing the threshold of the surface code [4,6-10], the true nature of the transition has been hard to assess due to the large Hilbert space that the code demands. Some criticism has also been raised by the use of simplified error models in these studies, since, for more traditional QEC schemes, error models that take into account correlations can substantially alter or even remove error thresholds [11-13]. In this paper we make significant progress on both issues. We consider more general bit-flip error models with and without disorder and correlations. We derive an exact mapping of a complete QEC quantum evolution with arbitrary syndrome onto a twodimensional Ising model with complex temperature. Exact results are then obtained for what we call the "one-cycle threshold." Our main conclusion is a positive one: A fidelity threshold exists in most cases, although its value is not universal, depending on the noise model. For a representative case, the analytical prediction for the threshold location based on the mapping is supported by Monte Carlo simulations.

The remainder of the paper is organized as follows. In Sec. II we discuss the difference between intrinsic and effective thresholds, which is crucial for the understanding of our results. The next two sections are mainly a review: Section III contains a concise description of the surface code and Sec. IV describes the code's evolution, syndrome extraction, and error correction within one cycle in very general terms. The description of our work begins in Sec. V, where some basic assumptions and definitions are provided and a suitable expression for the fidelity is presented. This is followed by a discussion in Sec. VI of decoding and the thermodynamic limit in the determination of the threshold. A realistic error model that induces bit-flip errors is introduced in Sec. VII and consists of an effective action involving single-qubit and twoqubit interaction terms. Using this error model and considering the full quantum evolution of the physical qubits, in Sec. VIII we map the fidelity calculation after one QEC cycle onto the evaluation of correlation functions of a two-dimensional Ising model. In Sec. IX we discuss several scenarios based on that mapping, including cases with homogeneous and inhomogeneous couplings. In Sec. X we present the result of Monte Carlo simulations of the fidelity threshold and confirm the analytical prediction based on the mapping for the homogeneouscoupling case. Conclusions are provided in Sec. XI

\section{INTRINSIC AND EFFECTIVE THRESHOLDS}

Two QEC strategies can be used for any stabilizer code. In the so-called active QEC, stabilizer operators are measured and, based on their syndromes, a recovery operation is chosen and implemented. In passive QEC, the physical qubits are subjected to a Hamiltonian that enforces an energy gap between the code word subspace and the rest of the Hilbert space of the physical qubits. Typically, the Hamiltonian consists of a sum over all stabilizer operators multiplied by a negative constant. Protection in this case requires neither measurements nor recovery operations. While the surface code introduced by Dennis and co-workers [4] is an example of active QEC, the original toric code of Kitaev [14] is its passive counterpart.

Now consider adding to the toric code a static perturbation that acts directly on the physical qubits and competes with the code's intrinsic Hamiltonian [15]. Several authors have shown that beyond a certain critical value of the perturbation's 
coupling constant, the toric Hamiltonian is no longer capable of protecting the code word subspace; topological order is completely lost and so is the spectral gap separating the ground-state subspace from the excited states [16-18]. In this context, it is clear that the critical value of the coupling constant provides an intrinsic threshold: Any perturbation larger than the threshold renders the code completely ineffective even when the code distance is increased. It is natural to assume that different types of perturbations will have distinct thresholds.

In this paper we extend the concept of intrinsic thresholds to active QEC. While for passive QEC the intrinsic threshold reveals itself as a clear-cut quantum phase transition, in active QEC the situation is a more subtle because the outcome of the QEC cycle depends on the syndromes, their decoding, and the recovery operation. Thus, it is natural to look for the intrinsic threshold in the most favorable situation, one that is not affected by a particular decoding strategy for codes. This happens in the case of a nonerror syndrome, when no recovery operation is recommended. For any other syndrome, the threshold must be less favorable because there can be a certain amount of uncertainty as to which recovery operation is more effective.

Within this approach, we distinguish two kinds of thresholds:

(1) an intrinsic one, which depends only on the interaction between the physical qubits and the environment and is independent of any decoding procedure;

(2) an effective one, which depends on the interaction between the physical qubits and the environment and on the decoding procedure.

This distinction is valid for any stabilizer code. The effective threshold is always equal or smaller than the intrinsic threshold. The effective threshold can always be increased by improving the decoding procedure until it reaches the intrinsic value. Thus, perfect decoding makes the effective threshold equal to the intrinsic one in the limit when the code distance goes to infinity.

In the context of the surface code, the existence of threshold has been demonstrated by associating it with a phase transition of a classical statistical spin model with quenched disorder [18]. The proof assumes that physical qubits are subjected to independent depolarizing noise sources, uncorrelated in time, with recovery from those errors requiring a syndrome decoding strategy. In this paper we go further and argue that, in general, an intrinsic threshold may exist independently of the syndrome decoding procedure adopted, being determined only by the error model. We show that an intrinsic threshold exists for a noise source where spatial correlations among physical qubits are induced by their interaction with a common environment. The threshold in this case is associated with a finite-temperature phase transition of a two-dimensional Ising model, with the coupling constant between physical qubits and the environment playing the role of the inverse temperature of the model.

\section{THE SURFACE CODE AND THE STABILIZER FORMALISM}

In a QEC stabilizer protocol, information is encoded into a much larger Hilbert space than the minimum space physically

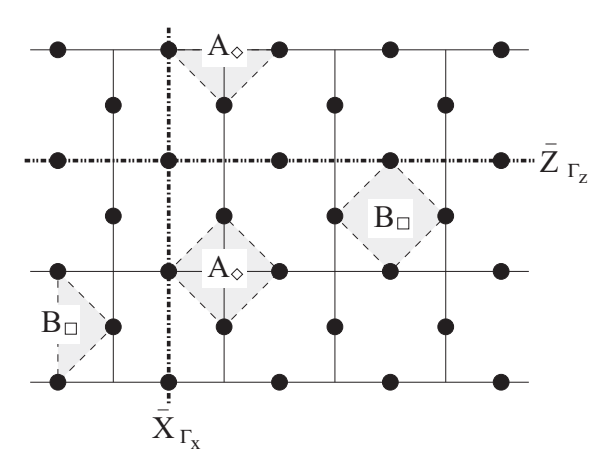

FIG. 1. Schematic representation of the surface code. The circles are physical qubits. The gray squares represent plaquettes $\left(B_{\square}\right)$ and stars $\left(A_{\diamond}\right)$. The product of single-qubit operators along the dash-dotted lines $\Gamma_{X}$ (vertical) and $\Gamma_{Z}$ (horizontal) define the logical operators $\bar{X}_{\Gamma_{X}}$ and $\bar{Z}_{\Gamma_{Z}}$, respectively.

required. Different sectors of this large Hilbert space are labeled by different values of observables associated to operators known as stabilizers. A judicial choice for the stabilizers can then be used to diagnose the most common type of error for a given quantum evolution. Based on the outcomes (syndromes) of measurements of stabilizer operators, a forceful return to the logical Hilbert space is performed.

The surface code consists of a two-dimensional array of qubits placed on the edges of a square lattice; see Fig. 1. These physical qubits can be implemented with Josephson junctions [19], cold atoms [20], trapped ions [21], Rydberg atoms [22], or semiconductor quantum dots [23]. The stabilizers of the code are the plaquette operators,

$$
B_{\square}=\prod_{\mathbf{i} \in \square} \sigma_{\mathbf{i}}^{z},
$$

and the star operators,

$$
A_{\diamond}=\prod_{\mathbf{i} \in \diamond} \sigma_{\mathbf{i}}^{x},
$$

where $\sigma_{i}^{x, z}$ are the Pauli operators $x$ and $z$ of qubit $i$. The logical operations are defined as strings of physical qubit operations,

$$
\bar{X}_{\Gamma_{X}}=\prod_{\mathbf{i} \in \Gamma_{X}} \sigma_{\mathbf{i}}^{x}
$$

and

$$
\bar{Z}_{\Gamma_{Z}}=\prod_{\mathbf{i} \in \Gamma_{Z}} \sigma_{\mathbf{i}}^{z},
$$

where $\Gamma_{Z}$ is a path that cuts through the lattice from left to right and $\Gamma_{X}$ is a path that goes from top to bottom [24]. Finally, the code words can be written as

$$
|\bar{\uparrow}\rangle=G\left|F_{z}\right\rangle
$$

and

$$
|\bar{\downarrow}\rangle=\bar{X}_{\Gamma_{X}} G\left|F_{z}\right\rangle
$$

where

$$
G=\frac{1}{\sqrt{2^{N_{\diamond}}}} \prod_{\diamond}\left(1+A_{\diamond}\right)
$$


and

$$
\left|F_{z}\right\rangle=|\uparrow\rangle_{\mathbf{1}} \otimes \cdots \otimes|\uparrow\rangle_{\mathbf{N}}
$$

is the ferromagnet state in the $z$ component of the physical qubits. Here $N_{\diamond}$ denotes the number of star operators on the lattice and $N$ is the total number of physical qubits. The product $\bar{X}_{\Gamma_{X}} G$ is independent of the particular choice of $\Gamma_{X}$ and uniquely defines $|\bar{\downarrow}\rangle$ (thus, hereafter we drop the path subscript in the logical operators). The logical space is a two-dimensional Hilbert space where all plaquettes and stars, when measured, return the value +1 ; by convention, this set is called the zero-charge sector. All other sets define sectors with nonzero charge and are characterized by the number of -1 syndrome values for the plaquette and star operators.

\section{QUANTUM EVOLUTION, SYNDROME, AND ERROR CORRECTION}

To make the discussion more concise, and without loss of generality for the case of bit-flip errors, let us assume that the system is initially prepared in the logical state $|\bar{\uparrow}\rangle$ and is not entangled with the environment $|e\rangle$,

$$
|\Psi(0)\rangle=|\bar{\uparrow}, e\rangle .
$$

The logical qubit and the environment evolve under a unitary evolution operator $U(\Delta)$ for a time $\Delta$,

$$
|\Psi(\Delta)\rangle=U(\Delta)|\Psi(0)\rangle .
$$

Since we are assuming only bit-flip errors, stars remain always with eigenvalue 1 under this evolution. On the other hand, plaquettes may have eigenvalue \pm 1 . Let us call $\{p\}$ a set of plaquettes that return a nontrivial syndrome, thus indicating an error. When the stabilizers are measured, the system's state vector is projected by the operator

$$
\mathcal{P}_{\{p\}}=\frac{1}{2^{N} \square} \prod_{\square^{\prime}}\left(1+B_{\square}\right) \prod_{p}\left(1-B_{p}\right),
$$

where $\square^{\prime}$ is the set of plaquettes with eigenvalue +1 . The decoding procedure associates with a syndrome a certain recovery operation. In the surface code, this corresponds to choosing a string $\mathcal{S}_{\{p\}}^{x}$ made of a product of $\sigma_{\mathrm{i}}^{x}$ operators that connect the plaquettes in $\{p\}$ pairwise or to the boundaries. This recovery operation results in the un-normalized state vector

$$
\begin{aligned}
\left|\Psi_{\mathrm{QEC}}\right\rangle_{\{p\}} & =\mathcal{S}_{\{p\}}^{x} \mathcal{P}_{\{p\}}|\Psi(\Delta)\rangle \\
& =\mathcal{S}_{\{p\}}^{x} \mathcal{P}_{\{p\}} U(\Delta)|\uparrow, e\rangle .
\end{aligned}
$$

A small but important simplification can be made: Notice that

$$
\begin{aligned}
\mathcal{S}_{\{p\}}^{x} \mathcal{P}_{\{p\}} & =\frac{1}{2^{N} \square} \mathcal{S}_{\{p\}}^{x} \prod_{\square}\left(1+B_{\square}\right) \prod_{p}\left(1-B_{p}\right) \\
& =\frac{1}{2^{N_{\square}}} \prod_{\square}\left(1+B_{\square}\right) \mathcal{S}_{\{p\}}^{x},
\end{aligned}
$$

where in the second line the product over plaquettes is unconstrained. Therefore,

$$
\left|\Psi_{\mathrm{QEC}}\right\rangle_{\{p\}}=\frac{1}{2^{N} \square} \prod_{\square}\left(1+B_{\square}\right) \mathcal{S}_{\{p\}}^{x} U(\Delta)|\uparrow, e\rangle .
$$

The presence of the projector $\prod_{\square}\left(1+B_{\square}\right)$ on the right-hand side of Eq. (14) implies that $\left|\Psi_{\mathrm{QEC}}\right\rangle$ contains only qubit states with all positive plaquettes; i.e., it can be represented by a superposition of the two logical states $|\bar{\uparrow}\rangle$ and $|\bar{\downarrow}\rangle$. The amplitude of each state depends on the choice of $\mathcal{S}_{\{p\}}$. A good choice will favor $|\bar{\uparrow}\rangle$.

Even though there is a large number of strings $\mathcal{S}_{\{p\}}^{x}$ compatible with the syndrome $\{p\}$, they can be sorted into two distinct classes related by the logical operator $\bar{X}$. The product $\mathcal{S}_{\{p\}}^{x} G$, implicit in the evolution of the logical qubit in Eq. (14), generates all possible strings within a class. Therefore, the particular choice of $\mathcal{S}_{\{p\}}^{x}$ to represent a class is irrelevant to the calculation of the fidelity of the code. After choosing a string $\mathcal{S}_{\{p\}}^{x}$, we can assign $\bar{X} \mathcal{S}_{\{p\}}^{x}$ to represent the elements of the other class.

Thus, if the recovery operation $\mathcal{S}_{\{p\}}^{x}$ brings the logical qubit state back to $|\bar{\uparrow}\rangle$, its counterpart $\bar{X} \mathcal{S}_{\{p\}}^{x}$ takes it to the state $|\bar{\downarrow}\rangle$. We can then write the (un-normalized) state vector at the end of the QEC cycle as

$$
\left|\Psi_{\mathrm{QEC}}\right\rangle_{\{p\}}=|\bar{\uparrow}\rangle\left\langle\bar{\uparrow}\left|\mathcal{S}_{\{p\}}^{x} U(\Delta)\right| \bar{\uparrow}, e\right\rangle+|\bar{\downarrow}\rangle\left\langle\bar{\uparrow}\left|\bar{X} \mathcal{S}_{\{p\}}^{x} U(\Delta)\right| \bar{\uparrow}, e\right\rangle .
$$

[Notice the partial contraction in the expectation values appearing on the right-hand side of Eq. (15); environmental degrees of freedom remain noncontracted.] In the case of a bad recovery operation, the roles of $\mathcal{S}_{\{p\}}^{x}$ and $\bar{X} \mathcal{S}_{\{p\}}^{x}$ are swapped. The fact that one cannot be completely sure of the efficacy of the recovery operation is the reason why the effective threshold is always equal to or smaller than the intrinsic threshold.

\section{ENVIRONMENT RESETTING AND THE ONE-CYCLE FIDELITY}

After one QEC cycle, the fidelity of the system comprising the physical qubits can be generally defined as

$$
\mathcal{F}_{\{p\}} \equiv \frac{\left|\left\langle\Psi(0) \mid \Psi_{\mathrm{QEC}}\right\rangle_{\{p\}}\right|^{2}}{\left\|\Psi_{\mathrm{QEC}}\right\|^{2}},
$$

where $\{p\}$ is the set of syndromes obtained. This definition implies that if the environment evolves to a state orthogonal to the ground state, the fidelity must go to zero regardless of the final state of the qubits.

Here we avoid this situation by assuming that the environment is reset to its ground state. It is physically reasonable to assume that the environment's excitations can be suppressed by some "cooling" mechanism (e.g., lowering the temperature, applying a polarizing field, etc). This assumption was previously discussed in Refs. [25,26]. Assuming this resetting of the environment at the end of the QEC cycle, we can rewrite Eq. (15) as

$$
\left|\Psi_{\mathrm{QEC}}\right\rangle_{\{p\}}=\mathcal{A}_{\{p\}}|\bar{\uparrow}, e\rangle+\mathcal{B}_{\{p\}}|\bar{\downarrow}, e\rangle,
$$

where we have introduced the amplitudes

$$
\mathcal{A}_{\{p\}}=\left\langle\uparrow, e\left|\mathcal{S}_{\{p\}}^{x} U(\Delta)\right| \bar{\uparrow}, e\right\rangle
$$

and

$$
\mathcal{B}_{\{p\}}=\left\langle\bar{\uparrow}, e\left|\bar{X} \mathcal{S}_{\{p\}}^{x} U(\Delta)\right| \bar{\uparrow}, e\right\rangle
$$


for each syndrome outcome characterized by the set $\{p\}$. As a result, after some simple manipulations [25], we can write

$$
\mathcal{F}_{\{p\}}=\frac{\left|\mathcal{A}_{\{p\}}\right|^{2}}{\left|\mathcal{A}_{\{p\}}\right|^{2}+\left|\mathcal{B}_{\{p\}}\right|^{2}} .
$$

Maintaining maximum fidelity at the end of the QEC cycle implies $\mathcal{F}_{\{p\}}=1$. Maximum loss of fidelity results from a complete uncertainty about the logical state, $\left|\mathcal{A}_{\{p\}}\right|=\left|\mathcal{B}_{\{p\}}\right|$, and, consequently, $\mathcal{F}_{\{p\}}=\frac{1}{2}$ [27].

When the coupling between the physical qubits and the environment is sufficiently weak, one can expand the evolution operator $U(\Delta)$ in terms of strings of operators $\sigma_{i}^{x}$ of increasing length. Each string represents a certain number of bit-flip events, with that number defining the length of the string. Looking at Eq. (18), one recognizes that the shortest string in the expansion that gives a nonzero contribution to $\mathcal{A}_{\{p\}}$ corresponds exactly to the string of $\sigma_{\mathbf{i}}^{x}$ operators in $\mathcal{S}_{\{p\}}$. Therefore, the order of the leading contribution to $\mathcal{A}_{\{p\}}$ is equal to the smallest possible length of $\mathcal{S}_{\{p\}}$. Similarly, the leading contribution to $\mathcal{B}_{\{p\}}$ has an order equal to the smallest possible length of $\bar{X} \mathcal{S}_{\{p\}}$. Therefore, in the weak-coupling limit, whenever $\mathcal{S}_{\{p\}}$ is shorter than $\bar{X} \mathcal{S}_{\{p\}}$ (namely, when it involves fewer bit flips), one expects $\left|\mathcal{A}_{\{p\}}\right|>\left|\mathcal{B}_{\{p\}}\right|$. In the strong-coupling limit, on the other hand, any perturbative expansion fails; in those circumstances, we expect $\mathcal{A}_{\{p\}}$ and $\mathcal{B}_{\{p\}}$ to have similar magnitudes on a finite lattice.

The dependence of $\mathcal{A}_{\{p\}}$ and $\mathcal{B}_{\{p\}}$ on the lengths of $\mathcal{S}_{\{p\}}$ and $\bar{X} \mathcal{S}_{\{p\}}$, respectively, give us a hint to the appropriate decoding procedure and thermodynamic limit we need to adopt in order to define a fidelity threshold.

\section{DECODING AND THE THERMODYNAMIC LIMIT}

The expression for the fidelity given in Eq. (20) is only valid under the assumption that the decoding of the syndromes is flawless, such that $\left|\mathcal{A}_{\{p\}}\right|>\left|\mathcal{B}_{\{p\}}\right|$. In practice, finding a recovery operation $\mathcal{S}_{\{p\}}^{x}$ that fulfills this inequality for any given syndrome set $\{p\}$ is a difficult task. A rich literature exists on decoding algorithms for the surface code; see, for instance, [4,28-30] and references therein, where a number of strategies have been proposed. Here we do not attempt to improve on the existing strategies. We offer instead a prescription where the inequality is always satisfied in the limit of infinite code distance (i.e., infinite lattice sizes). As we argue below, when the inequality holds, the fidelity threshold obtained from from Eq. (20) is equal to the intrinsic threshold. However, since it is not possible to guarantee that the decoding is flawless in practice, at times we will have $\left|\mathcal{A}_{\{p\}}\right|<\left|\mathcal{B}_{\{p\}}\right|$. Therefore, an effective fidelity threshold that takes into account the possibility of flawed recovery operations should always be smaller than the intrinsic one.

Our prescription for enforcing $\left|\mathcal{A}_{\{p\}}\right|>\left|\mathcal{B}_{\{p\}}\right|$ begins by recognizing that the amplitudes $\mathcal{A}_{\{p\}}$ and $\mathcal{B}_{\{p\}}$ are functions of the lattice size used to encode the logical qubit. Therefore, it is important to define what we call the thermodynamic limit. Let us start with a fixed and finite set $\{p\}$ containing an even number of plaquettes and assume that the limit is taken by constructing a sequence of lattices of increasing size. Consider the smallest lattice that can contain the set $\{p\}$ as the initial element of the sequence. The next element in the sequence is constructed from the previous one by adding rows and columns to all four boundaries of the lattice. As a consequence, the distance from any plaquette in $\{p\}$ to the boundaries increases with increasing lattice sizes. We now choose a string $\mathcal{S}_{\{p\}}^{x}$ made of a product of $\sigma_{i}^{x}$ operators that connect pairwise the plaquettes in $\{p\}$ without reaching the boundaries. By this choice, the string operator $\bar{X} \mathcal{S}_{\{p\}}$ used in the computation of $\mathcal{B}_{\{p\}}$ always reaches the boundaries, while $\mathcal{S}_{\{p\}}^{x}$ does not. As the lattice grows, the length of $\bar{X} \mathcal{S}_{\{p\}}$ surpasses that of $\mathcal{S}_{\{p\}}^{x}$. As described in Sec. $\mathrm{V}$, in the weak-coupling limit, $\mathcal{B}_{\{p\}}$ is strongly suppressed in comparison to $\mathcal{A}_{\{p\}}$. Thus, our choice of $\mathcal{S}_{\{p\}}^{x}$ ensures that, in the limit of infinite lattice size, the inequality $\left|\mathcal{A}_{\{p\}}\right|>\left|\mathcal{B}_{\{p\}}\right|$ is satisfied.

The prescription needs to be slightly modified when $\{p\}$ contains an odd number of plaquettes. After connecting all but one plaquette with strings pairwise, we connect the unpaired plaquette (presumably the most remote one) by a string to the closest boundary. We then keep that boundary fixed and construct the sequence of lattices by adding columns to the left and right boundaries but adding rows only to the opposite boundary. Thus, the one string in $\mathcal{S}_{\{p\}}^{x}$ reaching a boundary will keep its length fixed, while the counterpart of that string in $\bar{X} \mathcal{S}_{\{p\}}$ will reach the opposite boundary with an increasing length. This guarantees that $\left|\mathcal{A}_{\{p\}}\right|>\left|\mathcal{B}_{\{p\}}\right|$ is also satisfied.

With this definition of the thermodynamic limit at hand, we define the one-cycle threshold for the surface code as the largest value of coupling between the physical qubits and the environment such that, in the thermodynamic limit, $\mathcal{F}_{\{p\}} \rightarrow$ 1 for any finite set $\{p\}$. In particular, the intrinsic threshold corresponds to the nonerror syndrome, namely, when $\{p\}$ is an empty set. A proof that an intrinsic threshold always exists for the error model defined in Sec. VII is given in the Appendix . Our prescription for the thermodynamic limit also allows us to show in Sec. VIII that, for an error model with only nearestneighbor qubit interactions, the fidelity threshold derived from Eq. (20) is independent of the syndrome set $\{p\}$ and thus equal to the intrinsic threshold.

In our prescription for the thermodynamic limit we first fix the syndrome set $\{p\}$ and then take the lattice size to infinity. Thus, the density of plaquettes with eigenvalues -1 tends to zero. This procedure is quite adequate for our goal of establishing the intrinsic threshold irrespective of the syndrome $\{p\}$, as we argue in Sec. IX.

An alternative definition of the thermodynamic limit, commonly used in numerical investigations using stochastic error models, would keep the density of plaquettes with eigenvalues -1 fixed as the lattice size is increased. Therefore, the set $\{p\}$ would be different and larger for each new lattice size and a recalculation of the amplitudes $\mathcal{A}_{\{p\}}$ and $\mathcal{B}_{\{p\}}$ would be required at each new step. Thus, in addition to being much harder to be analyzed, such a prescription does not guarantee that the inequality $\left|\mathcal{A}_{\{p\}}\right|>\left|\mathcal{B}_{\{p\}}\right|$ is satisfied in the thermodynamic limit. Therefore, it is not suitable for determining a fidelity threshold from Eq. (20).

\section{THE ERROR MODEL}

In realistic implementations, the physical qubits interact with a variety of environmental degrees of freedom [31]. For instance, frequently one cannot neglect the interaction of qubits 
with bosonic environments [12]. These can come directly from phonons and electromagnetic fluctuations generated by electronic components or indirectly from interactions with spin or charge impurities. It is also possible that imperfections in qubit design cause spurious coupling between single-qubit states. Finally, it is also possible that qubits couple to a spin (or pseudospin) bath. For these cases and others, the effect of a time evolution under the influence of the environment can be recast as an effective action for the physical qubits by integrating out the environmental degrees of freedom. Thus, at the end of a QEC cycle, an effective evolution operator of the form

$$
U_{\text {eff }}=\langle e|U(\Delta)| e\rangle=e^{-H_{\text {eff }}}
$$

can be derived and employed in the calculation of matrix elements and probability amplitudes involving physical qubits. The exact form of $H_{\text {eff }}$ depends on the particular type of interaction and the nature of the physical qubits and the environment. Here we consider the two-term expression

$$
H_{\text {eff }}\left(\left\{\sigma_{\mathbf{i}}^{x}\right\}\right)=\sum_{\mathbf{i}} h_{\mathbf{i}} \sigma_{\mathbf{i}}^{x}+\sum_{\mathbf{i} \neq \mathbf{j}} J_{\mathbf{i}, \mathbf{j}} \sigma_{\mathbf{i}}^{x} \sigma_{\mathbf{j}}^{x} .
$$

The parameters $h_{\mathbf{i}}$ and $J_{\mathrm{ij}}$ incorporate environmental fields and qubit-qubit interactions, respectively, and can be either real or imaginary numbers. Their magnitudes set the strength of the coupling between physical qubits and the environment. This form is exact for qubits coupled linearly to free bosonic baths and local fields [26]. We note that $J_{\mathrm{ij}}$ can also represent direct interactions between qubits that are not environment mediated.

\section{MAPPING ONTO AN UNCONSTRAINED ISING MODEL}

Let us write expressions for the amplitudes $\mathcal{A}_{\{p\}}$ and $\mathcal{B}_{\{p\}}$ as sums over configurations of the variables $\left\{\sigma_{i}^{x}\right\}$. We begin by replacing each state $|\uparrow\rangle_{\mathbf{i}}$ in Eq. (8) by $\frac{1}{\sqrt{2}}\left(|+\rangle_{\mathbf{i}}+|-\rangle_{\mathbf{i}}\right)$, where $\hat{\sigma}_{\mathbf{i}}^{x}| \pm\rangle_{\mathbf{i}}= \pm| \pm\rangle_{\mathbf{i}}$. Introducing the notation $\hat{\sigma}_{\mathbf{i}}^{x}\left|\sigma_{\mathbf{i}}\right\rangle=\sigma_{\mathbf{i}}\left|\sigma_{\mathbf{i}}\right\rangle$, we have

$$
|\bar{\uparrow}\rangle=\sum_{\sigma}^{\prime}|\sigma\rangle
$$

up to a normalization factor. Here $\sigma$ stands for $\left(\sigma_{1}, \ldots, \sigma_{\mathbf{N}}\right)$. The sum in Eq. (23) is restricted to the configurations $\sigma$ that satisfy the constraint $A_{\diamond}=+1$ for all stars (i.e., vertices) in the lattice. We substitute Eq. (23) in the definitions of the amplitudes $\mathcal{A}_{\{p\}}$ and $\mathcal{B}_{\{p\}}$ [Eqs. (18) and (19)] and use Eq. (21) to arrive at

$$
\mathcal{A}_{\{p\}}=\sum_{\sigma}{ }^{\prime} \mathcal{S}_{\{p\}}^{x} e^{-H_{\mathrm{eff}}(\sigma)}
$$

and

$$
\mathcal{B}_{\{p\}}=\sum_{\sigma}{ }^{\prime} \bar{X} \mathcal{S}_{\{p\}}^{x} e^{-H_{\text {eff }}(\sigma)} .
$$

The operator $S_{\{p\}}^{x}$ now represents a string of variables $\sigma_{\mathbf{i}}$ compatible with the syndrome represented by the set of plaquettes $\{p\}$.

The sums in Eqs. (24) and (25) are very difficult to evaluate (see Ref. [25]). Below, we provide an exact solution to this problem for a particular but significant case.

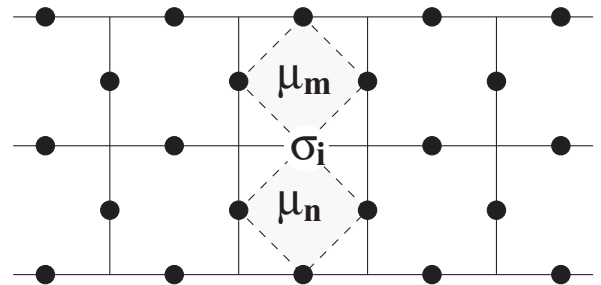

FIG. 2. Qubit variable $\sigma_{\mathbf{i}}$ and corresponding plaquette variables $\mu_{\mathbf{m}}$ and $\mu_{\mathbf{n}}$.

\section{Nearest-neighbor correlations}

Let us consider the case where $J_{\mathrm{ij}}$ describes only nearestneighbor interactions. To overcome the restriction in the sums in Eqs. (24) and (25), we introduce plaquette variables $\left\{\mu_{\mathbf{m}}\right\}$, with $\mu_{\mathbf{m}}= \pm 1$, such that $\sigma_{\mathbf{i}}=\mu_{\mathbf{m}} \mu_{\mathbf{n}}$ (see Fig. 2). The subscript $\mathbf{i}$ refers to the physical qubit $\mathbf{i}$, while $\mathbf{m}$ and $\mathbf{n}$ indicate the plaquettes sharing the link i. Even though the variables $\left\{\mu_{\mathbf{m}}\right\}$ can be positive or negative, they automatically satisfy the constraint of positive star eigenvalues. This parameterization is well known in the lattice gauge field literature [32]. Thus, starting from the error model defined in Eq. (22) and introducing these new variables, it is straightforward to show that, for nearest-neighbor qubits $\mathbf{i}$ and $\mathbf{j}$ in the bulk (i.e., not at the top or bottom edges),

$$
h_{\mathbf{i}} \sigma_{\mathbf{i}}^{x} \longrightarrow h_{\mathbf{i}} \mu_{\mathbf{m}} \mu_{\mathbf{n}}
$$

and

$$
J_{\mathbf{i j}} \sigma_{\mathbf{i}}^{x} \sigma_{\mathbf{j}}^{x} \longrightarrow J_{\mathbf{i j}} \mu_{\mathbf{u}} \mu_{\mathbf{v}},
$$

where the plaquettes $\mathbf{u}$ and $\mathbf{v}$ are next-to-nearest neighbors containing the links $\mathbf{i}$ and $\mathbf{j}$, respectively [see Fig. 3(a)]. Notice that the same product $\mu_{\mathbf{u}} \mu_{\mathbf{v}}$ appears again when we consider the contribution from the other pair of nearest-neighbor links $\mathbf{i}^{\prime}$ and $\mathbf{j}^{\prime}$ belonging to these plaquettes. Thus, we can define $\tilde{h}_{\mathbf{m n}}=h_{\mathbf{i}}$ such that

$$
\sum_{\mathbf{i} \in \text { bulk }} h_{\mathbf{i}} \sigma_{\mathbf{i}}^{x}=\sum_{\langle\mathbf{m}, \mathbf{n}\rangle} \tilde{h}_{\mathbf{m n}} \mu_{\mathbf{m}} \mu_{\mathbf{n}},
$$

(a)

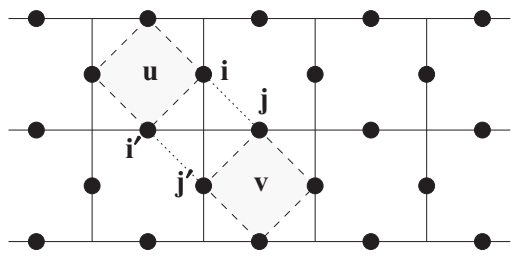

(b)

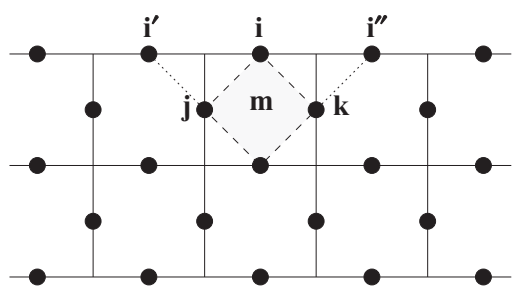

FIG. 3. (a) Links and plaquettes involved in Eq. (27). (b) Links and plaquette involved in Eq. (32). 
as well as $\tilde{J}_{\mathbf{u v}}=J_{\mathbf{i j}}+J_{\mathbf{i}^{\prime} \mathbf{j}^{\prime}}$ such that

$$
\sum_{\langle\mathbf{i}, \mathbf{j}\rangle \in \text { bulk }} J_{\mathbf{i j}} \sigma_{\mathbf{i}}^{x} \sigma_{\mathbf{j}}^{x}=\sum_{\langle\langle\mathbf{u}, \mathbf{v}\rangle\rangle} \tilde{J}_{\mathbf{u v}} \mu_{\mathbf{u}} \mu_{\mathbf{v}},
$$

where $\langle\mathbf{m}, \mathbf{n}\rangle$ are nearest-neighbor plaquettes and $\langle\langle\mathbf{u}, \mathbf{v}\rangle\rangle$ are next-to-nearest neighbors. The new parameters are functions of $h_{\mathbf{i}}$ and $J_{\mathbf{i j}}$. Notice that for homogeneous fields and couplings in the bulk, $h_{\mathbf{i}}=h$ and $J_{\mathbf{i j}}=J$, we get $\tilde{h}_{\mathbf{m n}}=\tilde{h}=h$ and $\tilde{J}_{\mathbf{u v}}=\tilde{J}=2 J$.

A little more work is needed to understand the constraint of positive stars at the bottom and top boundaries (see Fig. 1). A star at these boundaries is formed by the product of three qubits; for instance, $A_{1}=\sigma_{1}^{x} \sigma_{2}^{x} \sigma_{3}^{x}=1$. One of these qubits belongs to the bulk and can be written in terms of plaquette variables, $\sigma_{2}^{x}=\mu_{1} \mu_{3}$. Therefore, we obtain $\mu_{1} \sigma_{1}^{x} \mu_{3} \sigma_{3}^{x}=1$ and, consequently, $\mu_{1} \sigma_{1}^{x}=\mu_{3} \sigma_{3}^{x}= \pm 1$. This can be repeated for all qubits at the boundary. As a result, the product of any pair $\mu_{\mathbf{m}} \sigma_{\mathbf{i}}^{x}$ at the boundaries is a constant \pm 1 (here $\mathbf{m}$ denotes the plaquette where the boundary link $\mathbf{i}$ is located). Thus, for sites at the top $t$ and bottom $b$ boundaries, we can write

$$
h_{\mathbf{i}} \sigma_{\mathbf{i}} \longrightarrow \alpha_{t(b)} h_{\mathbf{i}} \mu_{\mathbf{m}}
$$

and

$$
J_{\mathbf{i j}} \sigma_{\mathbf{i}} \sigma_{\mathbf{j}} \longrightarrow \alpha_{t(b)} J_{\mathbf{i j}} \mu_{\mathbf{n}},
$$

where $\alpha_{t(b)}= \pm 1$ and $\mathbf{n}$ is the nearest-neighbor plaquette to $\mathbf{m}$ that contains the link $\mathbf{j}$. Similarly to the bulk case, pairwise interactions between a qubit at the boundary and another in the bulk contribute twice to terms containing solely one plaquette variable. As a result,

$$
\sum_{\mathbf{i} \in t(b)} h_{\mathbf{i}} \sigma_{\mathbf{i}}^{x}+\sum_{\langle\mathbf{i}, \mathbf{j}\rangle, \mathbf{i} \in t(b)} J_{\mathbf{i j}} \sigma_{\mathbf{i}}^{x} \sigma_{\mathbf{j}}^{x}=\alpha_{t(b)} \sum_{\mathbf{m} \in t(b)} \tilde{h}_{\mathbf{m}} \mu_{\mathbf{m}},
$$

where $\tilde{h}_{\mathbf{m}}=h_{\mathbf{i}}+J_{\mathbf{i}^{\prime} \mathbf{j}}+J_{\mathbf{i}^{\prime \prime} \mathbf{k}}$. Here $\mathbf{i}^{\prime}$ and $\mathbf{i}^{\prime \prime}$ are the next-tonearest neighboring links to $\mathbf{i}$ along the edge and $\mathbf{j}$ and $\mathbf{k}$ are links belonging to the same plaquette where $\mathbf{i}$ is located [see Fig. 3(b)].

Combining Eqs. (28), (29), and (32), we obtain

$$
H_{\text {eff }}\left(\left\{\mu_{\mathbf{m}}\right\}\right)=\sum_{\langle\mathbf{m}, \mathbf{n}\rangle} \tilde{h}_{\mathbf{m n}} \mu_{\mathbf{m}} \mu_{\mathbf{n}}+\sum_{\langle\langle\mathbf{u}, \mathbf{v}\rangle\rangle} \tilde{J}_{\mathbf{u} \mathbf{v}} \mu_{\mathbf{u}} \mu_{\mathbf{v}} .
$$

Thus, we can completely eliminate the qubit variables.

We can now go back to Eqs. (24) and (25) and switch the restricted sums over qubit variables $\sigma$ to unrestricted sums over plaquette variables $\left\{\mu_{\mathbf{m}}\right\}$. There are two distinct situations to consider, depending on the number of plaquettes in the syndrome.

For syndromes with an even number of plaquettes, the choice of strings in $S_{\{p\}}^{x}$ will involve no link at the top or bottom boundaries. This is because, in our thermodynamic limit, when the boundaries became infinitely far apart but the plaquettes are confined, this choice provides terms with a finite order in the coupling constants $h_{\mathbf{i}}$ and $J_{\mathbf{i j}}$; other choices will lead to infinite-order terms. Therefore, we can write

$$
\mathcal{S}_{\{p\} \text { even }}^{x} \longrightarrow \prod_{\mathbf{m} \in\{p\}} \mu_{\mathbf{m}}
$$

and

$$
\bar{X} \mathcal{S}_{\{p\} \text { even }}^{x} \longrightarrow \alpha_{b} \alpha_{t} \prod_{\mathbf{m} \in\{p\}} \mu_{\mathbf{m}},
$$

since any logic operator $\bar{X}$ will link top to bottom boundaries.

For syndromes with an odd number of plaquettes, at least one string in $S_{\{p\}}^{x}$ will have to end in one of the boundaries. By applying the operator $\bar{X}$ one generates a string ending on the opposite boundary. Then,

$$
\mathcal{S}_{\{p\} \text { odd }}^{x} \longrightarrow \alpha_{t(b)} \prod_{\mathbf{m} \in\{p\}} \mu_{\mathbf{m}}
$$

and

$$
\bar{X} \mathcal{S}_{\{p\} \text { odd }}^{x} \longrightarrow \alpha_{b(t)} \prod_{\mathbf{m} \in\{p\}} \mu_{\mathbf{m}} .
$$

[If the unpaired plaquette is linked to the top boundary by $\mathcal{S}_{\{p\}}^{x}$, then one picks the subscript $t$ on the right-hand side of Eq. (36). The opposite goes for Eq. (37).]

To obtain new expressions for the amplitudes $\mathcal{A}_{\{p\}}$ and $\mathcal{B}_{\{p\}}$ in terms of the variables $\left\{\mu_{\mathbf{m}}\right\}, \alpha_{b}$, and $\alpha_{t}$, one inserts Eqs. (34) and (35) or Eqs. (36) and (37) into Eqs. (24) and (25) and sums over all configurations of the variables $\alpha_{t}, \alpha_{b}$, and $\left\{\mu_{\mathbf{m}}\right\}$. In order to simplify the result, we introduce the (un-normalized) correlation function

$$
C_{\{p\}}\left(\alpha_{b}, \alpha_{t}\right)=\sum_{\left\{\mu_{\mathbf{m}}\right\}}\left(\prod_{\mathbf{k} \in\{p\}} \mu_{\mathbf{k}}\right) e^{-\tilde{H}\left(\left\{\mu_{\mathbf{m}}\right\} ; \alpha_{t}, \alpha_{b}\right)},
$$

where

$$
\tilde{H}\left(\left\{\mu_{\mathbf{m}}\right\} ; \alpha_{t}, \alpha_{b}\right)=H_{\text {eff }}\left(\left\{\mu_{\mathbf{m}}\right\}\right)+H_{\text {bound }}\left(\left\{\mu_{\mathbf{m}}\right\} ; \alpha_{t}, \alpha_{b}\right)
$$

and

$$
H_{\text {bound }}\left(\left\{\mu_{\mathbf{m}}\right\} ; \alpha_{t}, \alpha_{b}\right)=\alpha_{t} \sum_{\mathbf{t}} \tilde{h}_{\mathbf{t}} \mu_{\mathbf{t}}+\alpha_{b} \sum_{\mathbf{b}} \tilde{h}_{\mathbf{b}} \mu_{\mathbf{b}} .
$$

Notice that since $H_{\text {eff }}$ contains only two-body interaction terms, a symmetry relation is satisfied,

$$
C_{\{p\}}\left(-\alpha_{t},-\alpha_{b}\right)=(-1)^{N_{p}} C_{\{p\}}\left(\alpha_{t}, \alpha_{b}\right),
$$

where $N_{p}$ is the number of plaquettes in $\{p\}$. Using Eqs. (34)-(37) in conjunction with Eqs. (38) and (41) allows us to obtain concise relations for the amplitudes $\mathcal{A}_{\{p\}}$ and $\mathcal{B}_{\{p\}}$. For $N_{p}$ even, we get

$$
\mathcal{A}_{\{p\}}^{\text {even }}=C_{\{p\}}(+,+)+C_{\{p\}}(+,-)
$$

and

$$
\mathcal{B}_{\{p\}}^{\text {even }}=C_{\{p\}}(+,+)-C_{\{p\}}(+,-) .
$$

For $N_{p}$ odd, there are two situations to consider. When the bottom is closest boundary to the most remote plaquette in $\{p\}$, we get

$$
\mathcal{A}_{\{p\}}^{\text {odd; bottom }}=C_{\{p\}}(+,+)-C_{\{p\}}(+,-)
$$

and

$$
\mathcal{B}_{\{p\}}^{\text {odd; bottom }}=C_{\{p\}}(+,+)+C_{\{p\}}(+,-),
$$

while when the closest boundary is the top one we get

$$
\mathcal{A}_{\{p\}}^{\text {odd top }}=C_{\{p\}}(+,+)+C_{\{p\}}(+,-)
$$


and

$$
\mathcal{B}_{\{p\}}^{\text {odd top }}=C_{\{p\}}(+,+)-C_{\{p\}}(+,-)
$$

\section{THE THRESHOLD AS A PHASE TRANSITION}

As argued in Sec. VI, our prescription of the thermodynamic limit guarantees that $\left|\mathcal{A}_{\{p\}}\right|>\left|\mathcal{B}_{\{p\}}\right|$ as the lattice size grows and therefore we can use Eq. (20) to evaluate the fidelity. Equations (42) to (47) are exact expressions, valid for any lattice size and for any finite set $\{p\}$, provided that the effective interaction between physical qubits involves only nearest neighbors. Thus, the behavior of $\mathcal{A}_{\{p\}}$ and $\mathcal{B}_{\{p\}}$ is completely determined by $\tilde{H}$ and its associated correlation function $C_{\{p\}}$. Since the boundary-field Ising model defined by $\tilde{H}$ has a finite-temperature critical point, the fidelity threshold can be understood as this phase transition point.

Let us now show that this interpretation is valid. To simplify the argument, suppose that either $\tilde{h}=0$ and $\tilde{J} \neq 0$ or $\tilde{h} \neq 0$ and $\widetilde{J}=0$, in which case the Ising model defined in Eq. (33) can always be transformed into a ferromagnetic model (when $\tilde{J}=0$ ) or two decoupled ferromagnetic models (when $\tilde{h}=0$ ) by an appropriate change in the signs of the the variables $\mu_{\mathbf{m}}$ belonging to one of the sublattices.

\section{A. $N_{p}$ even}

Consider the case where $N_{p}$ is even. For temperatures above the critical value (i.e., for small-enough coupling constants), the $\left\{\mu_{\mathbf{m}}\right\}$ spin system of Eq. (33) is in a disordered (paramagnetic) phase. In the thermodynamic limit, because both boundaries become infinitely distant from the plaquettes in $\{p\}, C_{\{p\}}$ will not depend on boundary fields $\alpha_{b}$ and $\alpha_{t}$, namely, $C_{\{p\}}\left(\alpha_{t}, \alpha_{b}\right) \rightarrow \sum_{\left\{\mu_{\mathbf{m}}\right\}}\left(\prod_{\mathbf{k} \in\{p\}} \mu_{\mathbf{k}}\right) e^{-H_{\text {eff }}\left(\left\{\mu_{\mathbf{m}}\right\}\right)}=$ $C_{\{p\}}(0,0)$. Notice that even though spatial correlations among the spins $\left\{\mu_{\mathbf{k}}\right\}$ in the set $\{p\}$ decay exponentially in space, they are finite even in the infinite-lattice limit because they are locked in their positions. Therefore, $\left|C_{\{p\}}(0,0)\right|>0$ and $\mathcal{B}_{\{p\}} \rightarrow 0$ independently of $\{p\}$. As a result, $\mathcal{F}_{\{p\}} \rightarrow 1$.

Conversely, for low temperatures (i.e., large-enough coupling constants), the spin system is in an ordered (ferromagnetic) phase. We can then distinguish two situations: (i) $\alpha_{b} \alpha_{t}=1$, when there is an even number of domain walls running parallel to the top and bottom boundaries; (ii) $\alpha_{b} \alpha_{t}=-1$, when the number of domain walls is odd. In both situations the domain walls are rather costly energetically (the cost scales with $N_{\text {col }}$, the number of columns in the lattice). Thus, for low-enough temperatures and $N_{\text {col }} \gg 1$, we can assume that $C_{\{p\}}( \pm, \pm)$ is governed by spin configurations with no domain wall, whereas $C_{\{p\}}( \pm, \mp)$ is governed by configurations with just one domain wall. In addition, since correlations decay exponentially in space in the ordered phase as well, $\left\langle\left\langle\prod_{\mathbf{k} \in\{p\}} \mu_{\mathbf{k}}\right\rangle\right\rangle \approx \prod_{\mathbf{k} \in\{p\}}\left\langle\left\langle\mu_{\mathbf{k}}\right\rangle\right\rangle$, where $\langle\langle\cdots\rangle\rangle=$ $\sum_{\left\{\mu_{\mathrm{m}}\right\}}(\cdots) e^{-\tilde{H}\left(\left\{\mu_{\mathrm{m}}\right\} ; \alpha_{t}, \alpha_{b}\right)}$, with each $\left\langle\left\langle\mu_{\mathbf{k}}\right\rangle\right\rangle= \pm 1$, depending on which side of the domain wall the site $\mathbf{k}$ is located. Therefore, since we have an even number of plaquettes in $\{p\}$, $C_{\{p\}}( \pm, \pm)>0$. For $C_{\{p\}}( \pm, \mp)$, on the other hand, because the domain wall can cut across the plaquettes in $\{p\}$ in many different ways with similar energy costs, this amplitude results from a sum of many similar terms with alternating signs.
$C_{\{p\}}( \pm, \mp)$ is strongly suppressed with respect to $C_{\{p\}}( \pm, \pm)$ and, as a result, $\mathcal{A}_{\{p\}} \approx \mathcal{B}_{\{p\}}$ and $\mathcal{F}_{\{p\}} \approx \frac{1}{2}$, independently of the location of the plaquettes in the set $\{p\}$, as long as they are in finite number.

\section{B. $N_{p}$ odd}

Let us now consider cases where $N_{p}$ is odd and assume that the most remote plaquette in $\{p\}$ is closer to the top boundary (it is straightforward to extend the discussion to the opposite situation).

For temperatures higher than the critical one, the spin system is in a disordered (paramagnetic) phase. In the thermodynamic limit, the bottom boundary will become infinitely distant to the plaquettes in $\{p\}$ and $C_{\{p\}}\left(\alpha_{t}, \alpha_{b}\right)$ will not depend on the boundary field $\alpha_{b}: C_{\{p\}}\left(\alpha_{t}, \alpha_{b}\right) \rightarrow C_{\{p\}}\left(\alpha_{t}, 0\right)$. As a result, from Eqs. (46) and (47) we see that $\mathcal{B}_{\{p\}} \rightarrow 0$, independently of $\{p\}$, while $\mathcal{A}_{\{p\}}$ takes a nonzero finite value determined by the residual spatial correlations between the spin variables $\left\{\mu_{\mathbf{k}}\right\}_{\mathbf{k} \in\{p\}}$. Therefore, $\mathcal{F}_{\{p\}} \rightarrow 1$.

The argument for the low-temperature limit follows closely that developed in Sec. IX when the number of plaquettes is even. In the ordered (ferromagnetic) phase, $C_{\{p\}}( \pm, \pm$ ) is governed by spin configurations with no domain walls. Then $C_{\{p\}}(+,+)>0$ and $C_{\{p\}}(-,-)>0$. The correlation functions $C_{\{p\}}( \pm, \mp)$, on the other hand, are dominated by spin configurations with a single domain wall running parallel to the top and bottom boundaries and result from a sum of terms with alternating signs with roughly the same energy costs. As a result, they are suppressed in magnitude in comparison to $C_{\{p\}}( \pm, \pm)$. Thus, using Eqs. (46) and (47), we concluded that $\mathcal{A}_{\{p\}} \approx \mathcal{B}_{\{p\}}$ and $\mathcal{F}_{\{p\}} \approx \frac{1}{2}$.

\section{Phase transition}

For both $N_{p}$ even and odd, the abrupt change in behavior of the correlation function $C_{\{p\}}$ as the critical temperature is crossed is what renders the transition from $\mathcal{F}_{\{p\}}=1$ to $\mathcal{F}_{\{p\}}=$ $\frac{1}{2}$ sharp and what defines the location of the threshold value for the coupling constants.

We stress that this transition is not the same as that originally discussed in Ref. [4], where the error model was a purely stochastic one with no correlations. By virtue of the stochastic nature of the errors, their problem mapped onto a spin glass on the Nishimori line. Instead, the transition we obtain bares close resemblance to that for the toric code in the presence of a transverse field $[16,17]$. For the error model we adopt, if the coupling constants in $\tilde{H}$ are homogeneous, the spin system $\left\{\mu_{\mathbf{m}}\right\}$ does not behave as a spin glass.

We now discuss in more detail the critical behavior of the boundary-field Ising model in some special situations and how that behavior affects the surface code threshold.

\section{Homogeneous coupling}

Consider $\tilde{J}_{\mathbf{u v}}=0$ and $\tilde{h}_{\mathbf{m n}}=h<0$ and real, corresponding to a single-qubit relaxation channel. In this case, Eq. (33) is reduced to the ferromagnetic square-lattice Ising model with a boundary field. In particular, $\mathcal{A}_{0}$ and $\mathcal{B}_{0}$ are determined by the partition function of this model [33]. It is known that the free energy has two different terms: a boundary and 


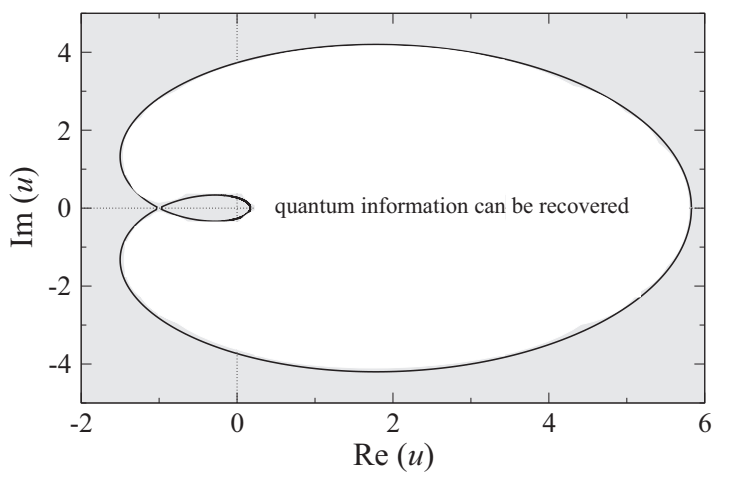

FIG. 4. Phase diagram of the Ising model with homogeneous complex coupling constant $h$ and its significance to the one-cycle QEC fidelity threshold. Here $u=e^{-4 h}$. In the gray regions quantum information cannot be recovered.

a bulk contribution. Although the boundary magnetization has a different exponent than the bulk one, the critical temperature for the ferromagnetic transition is defined by the bulk transition temperature [34], $\left|h_{\text {critical }}\right|=\ln (1+\sqrt{2}) / 2$. In the high-temperature paramagnetic phase, $|h|<\left|h_{\text {critical }}\right|$, the direction of the boundary fields is irrelevant. Hence, in this fully $Z_{2}$ symmetric phase, we find that $\mathcal{B}_{0} \rightarrow 0$ in the thermodynamic limit. Below the critical temperature, $|h|>\left|h_{\text {critical }}\right|$, the boundary fields explicitly break the $Z_{2}$ symmetry, leading to two distinct values for $\mathcal{B}_{0}$ when $\alpha_{t}=\alpha_{b}$ and $\alpha_{t} \neq \alpha_{b}$. In this phase, $\mathcal{B}_{0} \neq 0$ in the thermodynamic limit and we find $\mathcal{F}_{0}<1$. The transition is exponentially sharp since the boundary free energy is proportional to the number of sites at the edge. For other syndromes, a very similar discussion can be made. In the thermodynamic limit, the transition to a regime where the code can correct happens simultaneously for all syndromes since the critical point is entirely controlled by $\tilde{H}_{\text {eff }}$.

This analysis can be extended to complex $h$ [35]. In this more general situation, the transition point between the $Z_{2}$ symmetric phase and the broken symmetry phase is defined by the curve $\sinh ^{2}\left(2 h_{\text {critical }}\right)=e^{-i \theta}$, where $\theta \in[0,2 \pi)$ [see Fig. 4]. For a purely dynamical problem, when $h$ is imaginary (e.g., when a uniform external magnetic field acts on the physical qubits), $h_{\text {critical }}= \pm i \pi / 4$. In this case the critical point corresponds to $p_{c}=1 / 2$, which is consistent with the code providing infinite protection in the thermodynamic limit.

Now consider $\tilde{h}_{\mathbf{m n}}=0$ and $\tilde{J}_{\mathbf{u v}}=\tilde{J}<0$. This case applies to qubits coupled linearly to a gapless bosonic bath $[25,26]$. The Hamiltonian in Eq. (33) can be broken into two independent square-lattice Ising models with nearestneighbor interactions. Hence, the discussion from the previous paragraph can be immediately applied. Note that $\tilde{J}$ is doubled with respect to its value for the physical qubit interactions; i.e., $\tilde{J}=2 J$.

\section{E. Random coupling}

Let $\tilde{J}_{\mathbf{u v}}=0$ and $\tilde{h}_{\mathbf{m n}}$ be real and random. The Harris criterion cannot be applied since, for the clean Ising model, the specific heat critical exponent vanishes and the model is marginal to disorder [36]. We therefore discuss some specific cases. If $\tilde{h}_{\mathbf{m n}}$ has the same sign for all bonds, we expect bond disorder to be perturbative and simply yield a transition temperature roughly given by the typical value of $\tilde{h}_{\mathbf{m n}}$. This can be put on firm ground by considering a simple toy model with two possible values for the bond, $\tilde{h}_{\mathbf{m n}}=h_{1}$ or $h_{2}$, with equal probability [37]. In this case the transition temperature is given by $\left(e^{h_{1}}-1\right)\left(e^{h_{2}}-1\right)=2$.

An interesting situation arises when there is bond dilution, namely, when some of the $\tilde{h}_{\mathbf{m n}}$ are equal to zero. In the case of a square lattice, the percolation threshold happens when half of the bonds are missing. Thus, if at least half of the qubits do not suffer the action of a local magnetic field $h_{\mathbf{i}}$, the probability of having an infinite cluster (i.e., strings of flipped qubits traversing the lattice) tends to zero in the thermodynamic limit and the $Z_{2}$ broken phase does not exist. The implication to QEC is that quantum information can always be recovered if at least half of the qubits do not interact with the environment during the QEC cycle. Thus, only a severe random event that affects most of the qubits during the cycle will lead to a failure in the protection. This can be relevant to the design of other planar codes as well (which can be engineered to have high percolation thresholds).

The scenario dramatically changes when we allow bonds with different sign [38]. A Gaussian distribution is likely a realistic assumption for this case [39], but most of the physics can already be discussed using the toy-model bond distribution $P(h, q)=q \delta\left(\tilde{h}_{\mathbf{m n}}-h\right)+(1-q) \delta\left(\tilde{h}_{\mathbf{m n}}+h\right)$. There are three renormalization group fixed points for this model in the $(h, q)$ plane [38] (see Fig. 5). On top of the well-known Nishimori line, there is an unstable fixed point, $\left(h_{N}, q_{N}\right)$, that separates the $Z_{2}$ broken phase from the unbroken phase. For $q<q_{N}$ and $h<h_{N}$ the physics is controlled by the stable fixed point of the model $\left(h_{c}, 0\right)$; hence, we fall back to the discussion of the homogeneous model and get the usual paramagneticferromagnetic transition. Conversely, for $q \geqslant q_{N}$ and $h>h_{N}$ the transition is controlled by the fixed point with $\left(\infty, q_{N}\right)$. Little is known about this fixed point, but it is believed that it separates the ferromagnetic phase from a spin glass phase at $h=\infty$. The existence of the $Z_{2}$ broken phase is not in

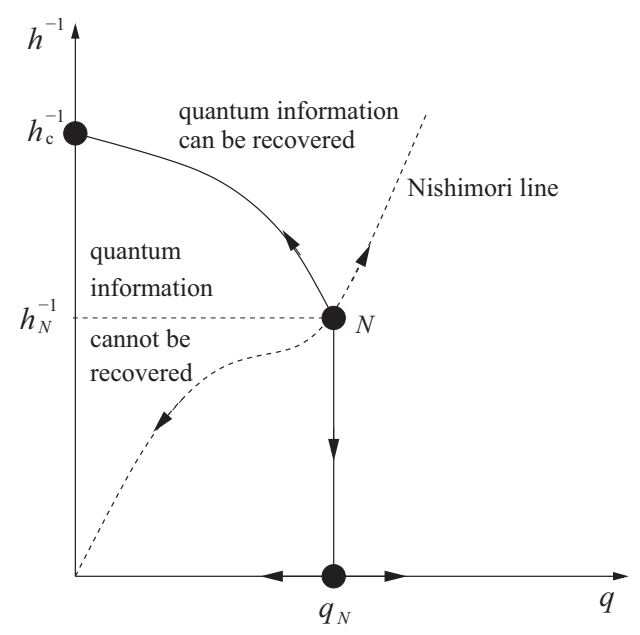

FIG. 5. Phase diagram of the Ising model with random bond sign and its significance to the one-cycle QEC fidelity threshold. 


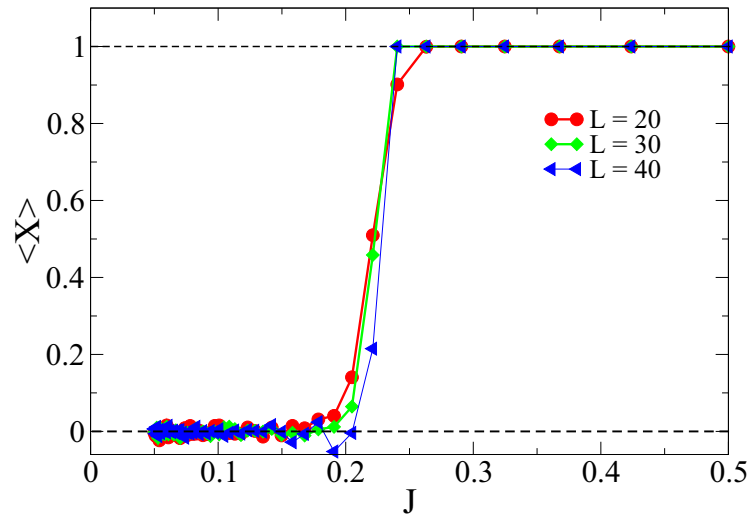

FIG. 6. (Color online) Ratio $\langle X\rangle=\left|\frac{\mathcal{B}}{\mathcal{A}}\right|$ evaluated through Monte Carlo sampling for three system sizes: $L=20$ (761 qubits, circles), $L=30$ (1741 qubits, diamonds), and $L=40$ (3121 qubits, triangles). The solid lines are guides for the eye.

question, but the nature of the unbroken phase, where we expect the fidelity to be higher, is not clear and needs further investigation.

Finally, let us consider $\tilde{h}_{\mathbf{m n}}=0$ and $\tilde{J}_{\mathbf{u v}}$ real and random: All the discussion from the previous paragraphs can be immediately transported to this case, the main difference being that there are two independent lattices. Hence, as a function of disorder or coupling strength, one can have two different transition temperatures and thus a more complicated threshold situation may arise.

\section{NUMERICAL SIMULATIONS}

Some of the results described above were independently confirmed by Monte Carlo simulations. Here we present the case of a constant and real $J_{\mathbf{i j}}=J$ for nearest neighbors, $h=0$, and nonerror syndromes. To insert the constraint $A_{\diamond}=1$ into the Metropolis algorithm, an alternative representation of the stabilizers was necessary. The stabilizer operator was rewritten as the product of an even number $m$ of logical operators such that their product is equivalent to the stabilizer, namely, $A_{\diamond}=$ $\bar{Z}_{\Gamma_{1}}, \ldots, \bar{Z}_{\Gamma_{2 m}}$ (see Ref. [40] for details of this formulation and more extensive numerical results). Working directly with the original spin (i.e., physical qubit) variables, we used this representation to numerically evaluate the amplitudes $\mathcal{A}_{0}$ and $\mathcal{B}_{0}$ and the ratio $\langle X\rangle=\left|\mathcal{B}_{0}\right| /\left|\mathcal{A}_{0}\right|$. Data for the case of nearest-neighbor interactions are shown in Fig. 6. Notice that the larger the lattice, the sharper becomes the transition. The mapping onto the unconstrained Ising model predicts that the critical coupling $J_{c}$ should be equal to half of that for a regular two-dimensional Ising system, namely, $J_{c} \approx 0.220$. This is in excellent agreement with the numerical value of $J_{c}=0.217$ obtained in our Monte Carlo simulations through a finite-size scaling analysis.

\section{CONCLUSIONS}

In this paper, we have argued that a stabilizer code, such as the surface code, when coupled to an environment, has an intrinsic fidelity threshold. This threshold can be determined by focusing on the nonerror syndrome sector after the physical qubits have evolved in time under an effective action intermediated by the environment. For other syndrome sectors, the decoding of the syndrome into a recovery operation limits the error correction capabilities of the code. As a result, the effective fidelity threshold should be smaller than the intrinsic threshold. To show that a threshold exists nevertheless for any syndrome, we provide a prescription where the decoding always works in the infinite lattice size limit. When bit-flip errors are considered, this prescription allows us to derive an expression for the fidelity of the surface code in terms of two amplitudes that involve expectation values of a constrained classical spin model.

For the case of an effective action involving at most nearest-neighbor interactions, we map the spin model onto an unconstrained Ising model with boundary fields. This mapping allows us to predict the exact value of the fidelity threshold for several important practical situations. We find that a fidelity threshold is almost always present, for both homogeneous and inhomogeneous noise sources, but the actual critical value of the coupling constant between qubit and environment depends on particular features of the model. Thus, the threshold is not universal. For the case of homogeneous coupling to the environment, the analytical prediction for the threshold location based on the exact mapping is confirmed by an independent Monte Carlo simulation.

When the effective interaction between physical qubits goes beyond nearest neighbors, the mapping no longer works, but the threshold can still be computed by direct numerical simulations of the constrained spin model. A recent work by two of the authors [40] shows that the threshold decreases with increasing interaction range, as originally predicted in Ref. [25].

\section{ACKNOWLEDGMENTS}

We are grateful to Robert Raussendorf for inspiring discussions. We thank José Hoyos Neto, David Poulin, and Barbara Terhal for useful conversations. E.N. was partially supported by INCT-IQ and CNPq. P.J. and E.R.M. were supported in part by the Office of Naval Research and the National Science Foundation through Grant No. CCF-1117241. I.S.T. acknowledges support from IARPA and PITP.

\section{APPENDIX: EXISTENCE OF AN INTRINSIC THRESHOLD}

Let us consider the case of a nonerror syndrome and assume that the environment is reset to its ground state at the end of the QEC cycle. The final state of the physical qubits is given by the state vector

$$
\left|\psi_{\mathrm{QEC}}\right\rangle_{0}=\mathcal{P}_{0} U_{\mathrm{eff}}|\uparrow\rangle
$$

where

$$
|\bar{\uparrow}\rangle=\frac{1}{\sqrt{2^{N_{\diamond}}}} \prod_{\diamond}\left(1+A_{\diamond}\right)\left|F_{z}\right\rangle,
$$

$\left|F_{z}\right\rangle$ is the ferromagnetic $z$ state, and the projector of positive stars and plaquettes is

$$
\mathcal{P}_{0}=\frac{1}{2^{N_{\diamond}} 2^{N_{\square}}} \prod_{\diamond}\left(1+A_{\diamond}\right) \prod_{\square}\left(1+B_{\square}\right) .
$$


Since "no errors" were identified, there is no need to apply a recovery operation: $\mathcal{S}_{0}^{x}=1$ (i.e., the identity operator).

Introducing the amplitudes

$$
\begin{aligned}
& \mathcal{A}_{0}=\left\langle\bar{\uparrow} \mid \psi_{\mathrm{QEC}}\right\rangle_{0}
\end{aligned}
$$

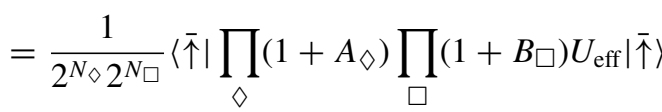

and

$$
\begin{aligned}
& \mathcal{B}_{0}=\left\langle\bar{\downarrow} \mid \psi_{\mathrm{QEC}}\right\rangle_{0}
\end{aligned}
$$

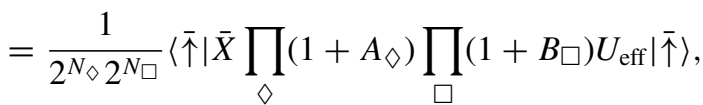

we can express the fidelity without any ambiguity as

$$
\mathcal{F}_{0}=\frac{\left|\mathcal{A}_{0}\right|^{2}}{\left|\mathcal{A}_{0}\right|^{2}+\left|\mathcal{B}_{0}\right|^{2}} .
$$

In order to evaluate the amplitudes, it is convenient to move to the $x$ basis $\{|s\rangle\}$. Since

$$
\left|F_{z}\right\rangle=\prod_{i=1}^{N}\left(\frac{|\uparrow\rangle_{x, i}+|\downarrow\rangle_{x, i}}{\sqrt{2}}\right)=\frac{1}{\sqrt{2^{N}}} \sum_{s}|s\rangle,
$$

we arrive at

$$
\mathcal{A}_{0}=\frac{1}{2^{N_{\square} 2^{N}}} \sum_{s}\left\langle s\left|\prod_{\diamond}\left(1+A_{\diamond}\right) \prod_{\square}\left(1+B_{\square}\right) U_{\mathrm{eff}}\right| s\right\rangle
$$

and

$$
\mathcal{B}_{0}=\frac{1}{2^{N_{\square} 2^{N}}} \sum_{s}\left\langle s\left|\bar{X} \prod_{\diamond}\left(1+A_{\diamond}\right) \prod_{\square}\left(1+B_{\square}\right) U_{\mathrm{eff}}\right| s\right\rangle,
$$

where we used that $\frac{1}{2^{N}}\left[\prod_{\diamond}\left(1+A_{\diamond}\right)\right]^{2}=\prod_{\diamond}\left(1+A_{\diamond}\right)$. Substituting $U_{\text {eff }}=e^{-H_{\text {eff }}}$ and recalling that, for bit-flip errors, $H_{\text {eff }}$ is diagonal in the $x$ basis, we can write

$$
\mathcal{A}_{0}=\frac{1}{2^{N_{\square} 2^{N}}} \sum_{s} e^{-E_{s}}\left\langle s\left|\prod_{\diamond}\left(1+A_{\diamond}\right) \prod_{\square}\left(1+B_{\square}\right)\right| s\right\rangle
$$

and

$$
\mathcal{B}_{0}=\frac{1}{2^{N_{\square}} 2^{N}} \sum_{s} e^{-E_{s}}\left\langle s\left|\bar{X} \prod_{\diamond}\left(1+A_{\diamond}\right) \prod_{\square}\left(1+B_{\square}\right)\right| s\right\rangle,
$$

where $H_{\mathrm{eff}}|s\rangle=E_{s}|s\rangle$.

At this point the sums over states $s$ contain all possible $x$ spin configurations of the physical qubits. However, the projectors in Eqs. (A10) and (A11) will restrict these configurations.
In order to proceed, we can explicitly write that any state $s$ that satisfies the projectors can be written in one of the two forms

$$
\begin{gathered}
\left|s_{1}\right\rangle=\prod_{j} B_{j}\left|F_{x}\right\rangle, \\
\left|s_{2}\right\rangle=\bar{Z}_{\gamma} \prod_{j} B_{j}\left|F_{x}\right\rangle,
\end{gathered}
$$

where $\left|F_{x}\right\rangle$ is the ferromagnetic state in the $x$ direction, $\bar{Z}_{\gamma}$ is a logical $z$ following a path $\gamma$, and $\prod_{j} B_{j}$ is a product over a set of plaquettes. In order for the basis $\left\{\left|s_{1}\right\rangle,\left|s_{2}\right\rangle\right\}$ to be complete, all possible products of plaquettes have to be used when generating the states $\left|s_{1}\right\rangle$. Notice that only one path $\gamma$ should be used for generating the states $\left|s_{2}\right\rangle$; summing over more than one path will render the basis overcomplete. In principle, the choice of $\gamma$ should not be important in the evaluation of the fidelity (choosing $\gamma$ amounts to choosing a gauge; for each state $\left|s_{1}\right\rangle$ there is a state $\left|s_{2}\right\rangle$ and different $\gamma$ 's just define different correspondences between those states).

Thus, the restricted sums can be explicitly written as

$$
\begin{aligned}
\mathcal{A}_{0} & =\frac{1}{2^{N}}\left(\sum_{s_{1}} e^{-E_{s_{1}}}\left\langle s_{1} \mid s_{1}\right\rangle+\sum_{s_{2}} e^{-E_{s_{2}}}\left\langle s_{2} \mid s_{2}\right\rangle\right) \\
& =\frac{1}{2^{N}}\left(\sum_{s_{1}} e^{-E_{s_{1}}}+\sum_{s_{2}} e^{-E_{s_{2}}}\right)
\end{aligned}
$$

and

$$
\begin{aligned}
\mathcal{B}_{0} & =\frac{1}{2^{N}}\left(\sum_{s_{1}} e^{-E_{s_{1}}}\left\langle s_{1}|\bar{X}| s_{1}\right\rangle+\sum_{s_{2}} e^{-E_{s_{2}}}\left\langle s_{2}|\bar{X}| s_{2}\right\rangle\right) \\
& =\frac{1}{2^{N}}\left(\sum_{s_{1}} e^{-E_{s_{1}}}-\sum_{s_{2}} e^{-E_{s_{2}}}\right) .
\end{aligned}
$$

Notice that $\left|\mathcal{A}_{0}\right| \geqslant\left|\mathcal{B}_{0}\right|$ always.

Now consider the infinite-lattice limit. When the spin model of Eq. (22) has a well-defined phase transition at a finite temperature, in the "high-temperature" (disordered) phase, which here corresponds to small coupling constant values, states of type $\left|s_{1}\right\rangle$ and $\left|s_{2}\right\rangle$ have the same "partition function," namely, $\sum_{s_{1}} e^{-E_{s_{1}}}=\sum_{s_{2}} e^{-E_{s_{2}}}$. As a result, $\mathcal{B}_{0}=0$ and $\mathcal{F}_{0}=1$ for coupling constant values below the critical point. In the "low-temperature" (ordered) phase, states $\left|s_{2}\right\rangle$ are energetically more costly than states $\left|s_{1}\right\rangle$ [25], resulting in $\left(\sum_{s_{1}} e^{-E_{s_{2}}}\right) /\left(\sum_{s_{2}} e^{-E_{s_{1}}}\right) \rightarrow 0$ in the thermodynamic limit. Therefore, for coupling constant values above the critical point, $\mathcal{B}_{0}=\mathcal{A}_{0}$ and $\mathcal{F}_{0}=1 / 2$.
[1] P. W. Shor, Phys. Rev. A 52, R2493 (1995).

[2] D. Gottesman, Ph.D. thesis, Caltech, 1997.

[3] S. B. Bravyi and A. Y. Kitaev, arXiv:quant-ph/9811052.

[4] E. Dennis, A. Kitaev, A. Landahl, and J. Preskill, J. Math. Phys. 43, 4452 (2002).

[5] A. G. Fowler, M. Mariantoni, J. M. Martinis, and A. N. Cleland, Phys. Rev. A 86, 032324 (2012).
[6] R. Raussendorf, J. Harrington, and K. Goyal, Ann. Phys. 321, 2242 (2006); New J. Phys. 9, 199 (2007); R. Raussendorf and J. Harrington, Phys. Rev. Lett. 98, 190504 (2007).

[7] D. S. Wang, A. G. Fowler, and L. C. L. Hollenberg, Phys. Rev. A 83, 020302(R) (2011).

[8] J. Ghosh, A. G. Fowler, and M. R. Geller, Phys. Rev. A 86, 062318 (2012). 
[9] A. G. Fowler, Phys. Rev. Lett. 109, 180502 (2012); arXiv:1310.0863; A. G. Fowler and J. M. Martinis, Phys. Rev. A 89, 032316 (2014).

[10] A. M. Stephens, Phys. Rev. A 89, 022321 (2014).

[11] R. Klesse and S. Frank, Phys. Rev. Lett. 95, 230503 (2005).

[12] E. Novais, E. R. Mucciolo, and H. U. Baranger, Phys. Rev. Lett. 98, 040501 (2007); Phys. Rev. A 82, 020303(R) (2010).

[13] J. Preskill, Quant. Inf. Comput. 13, 0181 (2013); H. K. Ng and J. Preskill, Phys. Rev. A 79, 032318 (2009).

[14] A. Yu. Kitaev, Ann. Phys. 303, 2 (2003).

[15] Several authors have shown that the passive two-dimensional toric code is thermally unstable [e.g., C. Castelnovo and C. Chamon, Phys. Rev. B 76, 184442 (2007)]. The instability is a general feature of two-dimensional topological systems with local interactions (O. Landon-Cardinal and D. Poulin, arXiv:1209.5750). This is because the strings of errors generated thermally can freely diffuse and wind around the torus, causing a logical error. The same problem does not arise in active QEC, which is the main focus of this paper, since it is possible to act on the state of the system and correct it before errors propagate far enough. In addition, we consider only zero temperature.

[16] S. Trebst, P. Werner, M. Troyer, K. Shtengel, and C. Nayak, Phys. Rev. Lett. 98, 070602 (2007).

[17] I. S. Tupitsyn, A. Kitaev, N. V. Prokof'ev, and P. C. E. Stamp, Phys. Rev. B 82, 085114 (2010).

[18] B. Terhal, arXiv:1302.3428.

[19] J. Clarke and F. K. Wilhelm, Nature (London) 453, 1031 (2008); D. P. DiVincenzo, Phys. Scr. T 137, 014020 (2009).

[20] I. Bloch, J. Dalibard, and W. Zwerger, Rev. Mod. Phys. 80, 885 (2008).

[21] D. Nigg et al., Science 345, 302 (2014).

[22] M. Saffman, T. G. Walker, and K. Mølmer, Rev. Mod. Phys. 82, 2313 (2010).

[23] M. A. Eriksson et al., Quant. Info. Proc. 3, 133 (2004); H. Bluhm et al., Nat. Phys. 7, 109 (2011).

[24] The allowed $\Gamma_{Z}$ paths can be obtained from that shown in Fig. 1 by pulling it up or down at the sites on horizontal edges. Similarly, allowed $\Gamma_{X}$ paths are generated from that shown in Fig. 1 by pulling it to left or to the right at the sites on horizontal edges.

[25] E. Novais and E. R. Mucciolo, Phys. Rev. Lett. 110, 010502 (2013).
[26] P. Jouzdani, E. Novais, and E. R. Mucciolo, Phys. Rev. A 88, 012336 (2013). Here avoid this situation by focusing on the zero-temperature case, when the bath does not evolve into a thermal mixture.

[27] Notice that the time evolution determined by Eq. (15) will always lead to a superposition between logical up and logical down states with nonzero amplitudes $A_{\{p\}}$ and $B_{\{p\}}$, respectively. The syndrome alone cannot distinguish between a logical $\bar{X}$ and the identity. Therefore, both amplitudes are always present, even after the syndrome and recovery. In the worst-case scenario, the bath would cause the magnitudes $\left|A_{\{p\}}\right|$ and $\left|B_{\{p\}}\right|$ to be equal, in which case the lowest possible fidelity is $1 / 2$, signaling a complete loss of information about the logical state. There is no situation where all amplitudes $A_{\{p\}}$ go to zero, unless the error operators have identical coupling constants and act uniformly on all qubits of the code simultaneously. However, in this case, the system is just doing a global logical state precession.

[28] G. Duclos-Cianci and D. Poulin, Phys. Rev. Lett. 104, 050504 (2010).

[29] S. Bravyi, M. Suchara, and A. Vargo, Phys. Rev. A 90, 032326 (2014).

[30] A. J. Ferris and D. Poulin, Phys. Rev. Lett. 113, 030501 (2014).

[31] P. C. E. Stamp and I. S. Tupitsyn, Chem. Phys. 296, 281 (2004); A. Morello, P. C. E. Stamp, and I. S. Tupitsyn, Phys. Rev. Lett. 97, 207206 (2006).

[32] A. M. Polyakov, Gauge Fields and Strings (Hardwood Academic, London, 1987).

[33] B. M. McCoy and T. T. Wu, Phys. Rev. 162, 436 (1967); D. B. Abraham, Phys. Rev. Lett. 44, 1165 (1980); H. Au-Yang and M. E. Fisher, Phys. Rev. B 21, 3956 (1980); A. Maciolek and J. Stecki, ibid. 54, 1128 (1996).

[34] L. Onsager, Phys. Rev. 65, 117 (1944).

[35] V. Matveev and R. Shrock, J. Phys. A: Math. Gen. 28, 1557 (1995).

[36] J. L. Jacobsen and J. Cardy, Nucl. Phys. B 515, 701 (1998).

[37] J. Cardy and J. L. Jacobsen, Phys. Rev. Lett. 79, 4063 (1997).

[38] A. Honecker, J. L. Jacobsen, M. Picco, and P. Pujol, in Proceedings of the NATO Advanced Research Workshop on Statistical Field Theories, edited by A. Cappelli and G. Mussardo (Kluwer Academic, Dordrecht, 2002).

[39] W. L. McMillan, Phys. Rev. B 29, 4026 (1984).

[40] P. Jouzdani and E. R. Mucciolo, Phys. Rev. A 90, 012315 (2014). 